\title{
Design and Modelling of a Micro Swimming Robot
}

Fei Yuan, School of Information of Science and Engineering, Chengdu University, Chengdu, China, yuanfei@cdu.edu.cn

Mohammad Hasan, School of Computing and Digital Technology , Staffordshire University, Stoke-on-Trent, UK, M.S.Hasan@staffs.ac.uk

Hongnian Yu, Dongguan Industry 4.0 Artificial Intelligence Laboratory, Dongguan University of Technology, Dongguan 523808, China; Department of Computing, Bournemouth University, Bournemouth, UK yu61150@IEEE.ORG

\begin{abstract}
The swimming micro-robot model starts from the discussion of the solution of Navier-Stokes equation. Fortunately, the micro-swimmers can be regarded as swimming in low Reynolds number flow which is dominated by Stoke equation. The research starts from a simple design which contains just one flagellum. After that, the whole swimmer is discussed, and then the swimmer with double flagella. These designs use a suitable model method and are discussed in dimensional details.
\end{abstract}

Keywords-Swimming micro-robot, low Reynolds number, Stoke equation, double flagella.

\section{INTRODUCTION}

Since the invention of industrial robots, the types of robots have increased, their performances have improved, and the fields of work have been expanding. The applications of the robot spread from the deep sea to the universe; especially in a variety of extreme environments where a human cannot access.

In the recent several decades, robotics research has begun to enter new fields of developments: giant systems (such as fully autonomous control of high artificial intelligence unmanned aerospace station) and micro-systems. Micro-robot generally refers to small-size as well as the small workload of the robot. With the development of Micro-ElectroMechanical Systems (MEMS) technology, micro-robot research has a wide range of application prospects and social needs e.g. biomedical, aerospace, defence, industry, agriculture, medical care etc. One kind of micro-robot is swimming micro-robot or micro swimmer which can move in a liquid environment. This kind of mobile micro-robot can enter a variety of liquid and spaces which are too small to work and explore for human beings.

Some applications of such robots include micro-satellite, micro-inertial navigation device, micro-instruments, distributed battlefield sensor network, the robot under narrow space and special operating conditions, car driving and security systems, agricultural genetic engineering, biochemical sensors etc.

Among these, the swimming pipeline micro-robot has become a popular research topic [1], [2].

Industrial piping micro-robots carry sensors and manipulators, go into the hazardous areas of non-removable piping systems e.g. space shuttles, missiles, nuclear power plants, or industrial micro-channels etc. to perform an inspection, repair cable routing and micro-cracks etc.

With the micro-robot further miniaturisation, it can also be applied to human body organs and blood vessels [3], for detection of lesions [4], [5], clear blood vessels [6], surgery [7] etc.

There are four kinds of micro-swimmer that employ different propelling methods: three-link flagellum, helix flagellum, flat wave flagellum and cilia as shown in Fig. 1.
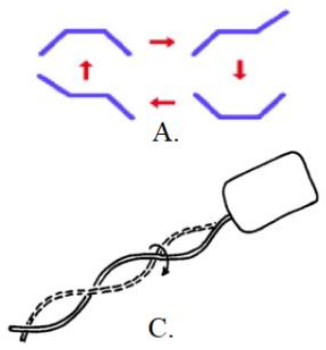

A. Three-link swimmer B. flat-wave flagella swimmer

C. helix flagella swimmer D. Cilia swimmer

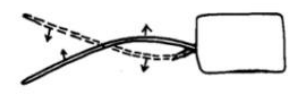

B.

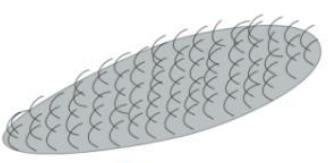

D.

Fig.1. Swimming model Categories

The most efficient propel method is helix propeller in many cases [8]. This research reviews existing helix propeller models and proposes a new double-helix propeller design which can improve the performance for better stability of axially moving. The rest of the paper is organised as follows. Section II describes the preliminaries and relevant works, section III presents the model of the proposed double-flagellum single body swimming robot, section IV investigates optimum strategies for the robot parameters using Matlab simulations and finally, section V draws some conclusions. 


\section{PRELIMINARIS AND RELEVANT WORKS}

The discussion about the hydrodynamic at low Reynolds number can be found in many pieces of research.

The theoretical background is low Reynolds number flow also known as Stokes flow [9].

The equation governing low Reynolds number flow is given below.

$$
\begin{aligned}
& \nabla p=\mu \nabla^{2} u+f \\
& \nabla \cdot u=0
\end{aligned}
$$

Here $\mathrm{u}$ is the velocity vector field, $\mathrm{p}$ is the pressure scalar field, $\mu$ is the dynamic viscosity, and $\mathrm{f}$ is the body forces acting on the fluid. This is known as the Stokes equation; a simplification of the Navier-Stokes equation and is correct only for $\operatorname{Re}=0$. The condition is hard to achieve but fortunately, the Stokes equation can be used as an approximate solution for $\mathrm{Re} \ll 1$. This kind of fluid is known as Stokes flow. Other names for this kind of flow include creeping flow and low Reynolds number flow. To swimming in this kind of flow, a system must utilise multiple degrees of freedom except for some specific conditions e.g. shear thickening and shear thinning (non-Newtonian) fluid [10].

There are two conditions that need to be satisfied to fabricate a realistic swimming micro-robot.

Firstly, energy should be transferred into a mechanical body to propel the robot.

Secondly, the propeller should not perform a time-reversible motion. Viscosity dominates the fluid dynamics at the micro-metre scale. This fact is discovered by the Scallop theory condition [11], it can only get a back and forward movement and always stay in original place whatever the oar is moving fast or slow because the flow is time-reversible. Although a swimmer can be found swimming by reciprocal motion [12], the scallop theorem still plays as an important role in low Reynolds number swimming [13].

The development of swimming micro-robot has two kinds of strategy: with a body and without a body. There are some designs that contain only tails and driven by the change of the outer magnetic field. To response to the future demands, this research adopts the design of tails with body.

\section{A. Design and Modelling of a Rigid Flagellum}

\section{1) Design of a rigid flagellum}

The design of the rigid flagella is derived from the shape of the microbial flagellum and the tail material is set to rigidity. Most microbes that rotate the flagellum are a left-handed spiral. The parameters to be considered are shown in Table I.

TABLE I. PARAMETERS IN Rigid Flagellum DESIGN

\begin{tabular}{|l|c|l|}
\hline \multicolumn{1}{|c|}{ Parameters } & Expression & typical range \\
\hline Helix radius & $\mathrm{R}$ & \\
\hline Pitch & $\lambda$ & $2 \mathrm{R}<\lambda<11 \mathrm{R}$ \\
\hline Length & $\mathrm{L}$ & $3 \lambda<\mathrm{L}<11 \lambda$ \\
\hline Pitch angle & $\theta$ & \\
\hline Contour Length & $\Lambda=\mathrm{L} / \cos \theta$ & \\
\hline Filament radius & $\mathrm{a}$ & \\
\hline
\end{tabular}

\section{2) Modelling of a rigid flagellum}

Firstly, the Reynolds number need to be considered. The typical Reynolds number for flagellum is $10^{-4}-10^{-2}$ from $R e=\rho \Omega R^{2} / \mu(\Omega$ is the rotation rate; $\mu$, the dynamic viscosity; and $\rho$, the fluid density). A rotating helical flagellum can produce forward thrust. The thrust $\mathrm{F}$ and torque $\tau$ have a relationship with flagellum' $\mathrm{s}$ axial velocity $\mathrm{U}$ and rotation rate $\Omega$ which is shown in (1)

$$
\left(\begin{array}{l}
F \\
\tau
\end{array}\right)=\left(\begin{array}{ll}
A & B \\
B & D
\end{array}\right)\left(\begin{array}{l}
U \\
\Omega
\end{array}\right)
$$

This $2 \times 2$ matrix is proposed by Purcell [14]. The equation shows that the propulsion thrust depends on the rotation torque and geometry parameters of the flagellum.

The values of A, B and D are shown in (2)-(4).

$$
\begin{array}{r}
A=2 \pi n R\left(\frac{\xi_{\|} \cos ^{2} \theta+\xi_{\perp} \sin ^{2} \theta}{\sin \theta}\right) \\
B=2 \pi n R^{2}\left(\xi_{\|}-\xi_{\perp}\right) \cos \theta \\
D=2 \pi n R^{3}\left(\frac{\xi_{\perp} \cos ^{2} \theta+\xi_{\|} \sin ^{2} \theta}{\sin \theta}\right)
\end{array}
$$

Here the values of $\xi_{\mid}$and $\xi_{\perp}$ are given by (5) and (6). 


$$
\begin{array}{r}
\xi_{\|}=\frac{4 \pi \eta}{\ln \left(\frac{0.36 \pi R}{a \sin \theta}\right)+\frac{1}{2}} \\
\xi_{\perp}=\frac{2 \pi \eta}{\ln \left(\frac{0.36 \pi R}{a \sin \theta}\right)}
\end{array}
$$

Here $R$ is helix amplitude, $\eta$ is viscosity and $a$ is filament radius.

The position of the flagella corresponding to these parameters is shown in Fig. 2.

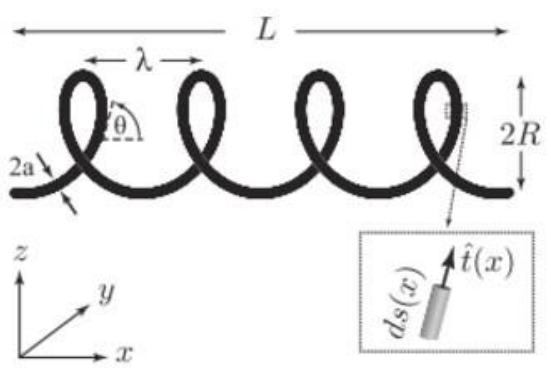

Fig. 2. Rigid Tail model

Another drag coefficient from Lighthill [15] is shown in (7) and (8) where $\lambda$ is the wavelength.

$$
\begin{aligned}
\xi_{\|} & =\frac{2 \pi \eta}{\ln \left(\frac{2 \lambda}{a}\right)-2.9} \\
\xi_{\perp} & =\frac{4 \pi \eta}{\ln \left(\frac{2 \lambda}{a}\right)-1.9}
\end{aligned}
$$

\section{B. Design and Modelling of a single Rigid Flagellum with a body}

\section{1) Design of a single rigid flagellum with a body}

The basic components of this design consist of a body, a spiral flagellum and a connecting part between the two. The connecting portion is seen as a hook that extends from the body and is attached to the spiral flagellum. The connecting part receives the torque from the body and conducts it to the spiral flagellum. The torque is the active component that drives the motion of the whole system.

The flagellum is a spiral structure that is rotated by the effect of the torque applied there. Specifically, the main part of the model is designed as an ellipsoid; the flagella portion is designed as a tubular spiral with a centerline; the connecting portion is designed as a conical spiral with a centerline, and the conical spiral centerline represents a hook connecting the main body to the flagella. The design is shown in Fig. 3 .

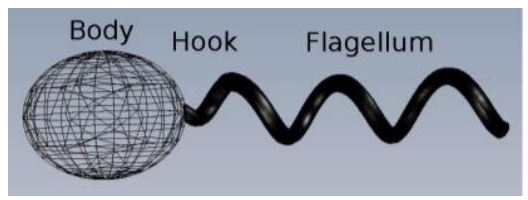

Fig. 3. The tail model with body

2) Modelling of a single rigid flagellum with a body

In propulsive matrix (1), the off-diagonal elements should be negative, $B<0$, for a right-handed spiral. The rotate direction of the torque needs to meet the condition of $\omega<0$ to drive the helix and push the system to the left, ie. $v>0$. The external force $F$ acting on the helix is negative, that is, toward the right side. The body part rotation reversed, that is, toward the right side. The body part rotation reversed, which means $\Omega<0$. Considering the above requirements, in order to meet the balance of force and torque on the central axis of the spiral, the following conditions must be met.

$$
\begin{aligned}
A_{0} v & =-A v-B \omega \\
D_{0} \Omega & =-B v-D \omega
\end{aligned}
$$


Since the motor itself is also rotating, its speed can be expressed as $\omega-\Omega$, which is the speed of the "rotor" connected to the flagella relative to the "stator" attached to the outer wall of the body, which is greater than $\omega$. Let the motor speed be set to $\Omega_{m}$ and derive the following equation

$$
v=-\frac{B D_{0}}{\left(A_{0}+A\right)\left(D_{0}+D\right)-B^{2}} \Omega_{m}
$$

From this, there are two steps approximation can be done.

Step 1, in some practical cases, $B^{2}$ is much smaller than $A D$. Because $B^{2}<<A D$, (11) can be well approximated by

$$
v=-\frac{B D_{0}}{\left(A_{0}+A\right)\left(D_{0}+D\right)} \Omega_{m}
$$

Step 2, the factor $D_{0} /\left(D_{0}+D\right)$ is just the ratio $\omega / \Omega_{m}$. Because the reverse rotation speed of the main body portion is much slower than the rotation speed of the flagellum, so the value of $D_{0} /\left(D_{0}+D\right)$ will be close to 1 . Therefore, the equation can further be approximated by

$$
v=-\frac{B}{A_{0}+A} \Omega_{m}
$$

Next, the efficiency is discussed. Firstly, the torque $\tau$ exerted by the motor on the flagellum is present in

$$
\tau=\frac{B^{2}-D\left(A_{0}+A\right)}{B} v
$$

Let the output power of the motor be $\tau \Omega_{m}$, and the minimum power required to push the entire structure move at the speed of $\mathrm{v}$ is $A_{0} v^{2}$. The ratio of $A_{0} v^{2}$ to $\tau \Omega_{m}$ is the definition of the propulsive efficiency $E$. With the relations above, the efficiency is present in

$$
\begin{aligned}
E & =\frac{A_{0} v^{2}}{\tau \Omega_{m}} \\
& =\frac{A_{0} D_{0} B^{2}}{\left[\left(A_{0}+A\right) D-B^{2}\right]\left[\left(A_{0}+A\right)\left(D_{0}+D\right)-B^{2}\right]}
\end{aligned}
$$

Using the same approximation steps, the efficiency can be derived in

$$
E=\frac{A_{0} B^{2}}{\left(A_{0}+A\right)^{2} D}
$$

\section{THE PROPOSED DESIGN OF DOUBLE FLAGELLA WITH BODY}

\section{A. Design of double rigid flagella with a body}

This design inspired from the structure of DNA. The moving velocity and efficiency may increase significantly by using this structure. The design contains a rigid body and 2 rigid tails connected to the body. The connection part, which consists of a hook coming out from the body and attaches to the two helical flagella, has at its base the rotor engine that drives the motion. The design is shown in Fig. 4.

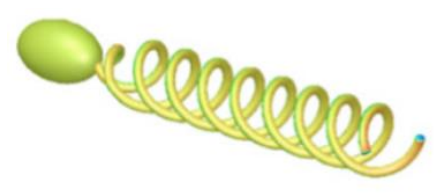

Fig. 4. Design 3 Double-tails with body

\section{B. Modelling of double rigid flagella with a body}

It can be noted that the flow around tails will move faster than a single tail. The axisymmetric flagella can make the system achieve more stable and reduce the tremble. It may achieve better performance. On the other hand, the flagella should be rigid one or elastic on with a slight degree of flexibility. For the soft flagella will bundling together when they start to rotate [16]. The velocity of the swimmer should have a bigger velocity that may approximately present by (17).

$$
v=-\frac{B D_{0}^{2}}{\left(A_{0}+A\right)\left(D_{0}+D\right)-B^{2}} \Omega_{m}
$$




\section{MODEL SHAPE PARAMETER OPTIMISATION}

This section investigates the effects of various parameters e.g. filament radius, helix amplitude etc. on the velocity and the effects of length of short axis on viscous resistance coefficients.

\section{A. Flagellum Shape optimization}

It can be noted that the whole shape of the flagellum is similar to a cylinder and a cone. The shape changes slightly when in motion. There are several different structures for the artificial flagellum. The condition of this theory is the low Reynolds number. It can be set for helix radio A is $6 \mathrm{~mm}$, the rotational speed of motor w is $0.1 \mathrm{~Hz}$. The environment parameters set as follow:

Dynamic viscosity $\mu=104 \mathrm{~N} \mathrm{~s} / \mathrm{m} 2$,

Density $\rho=9.55 \times 102$,

It can be calculated for $\operatorname{Re} \approx 10^{-5}$, the environment is similar to Stoke flow. Under this condition, the inner force is much smaller than the viscous force so that the resistance force theory can be applied to calculate the thrust force. It needs to define some parameters for analysing the thrust force and torque of artificial flagellum. The pitch of the artificial flagellum can be fixed when designing this conical-helix.

The optimisation of the shape of a helical flagellum is important for improving swimming performance independent of the input field.

The designed model consists of a body, a helical flagellum, and the junction connecting them. The engine is modelled by a torque. The parameters involved in the model are given below.

Number of pitch $n$,

Helix amplitude R,

wavelength $\lambda$,

length $\mathrm{L}$,

pitch angle $\theta$,

filament radius a.

There are some relationships between these parameters, for example, $L=\lambda \mathrm{n}, \tan \theta=\mathrm{R} / \lambda$, so the following four independent parameters are discussed.

- Filament radius a.

- Helix amplitude R,

- Helix wave number $\mathrm{n}$

- Wavelength $\lambda$,

Fig. 5, Fig. 6, Fig. 7, Fig. 8 show the relationship between above four parameters and the robot velocity.

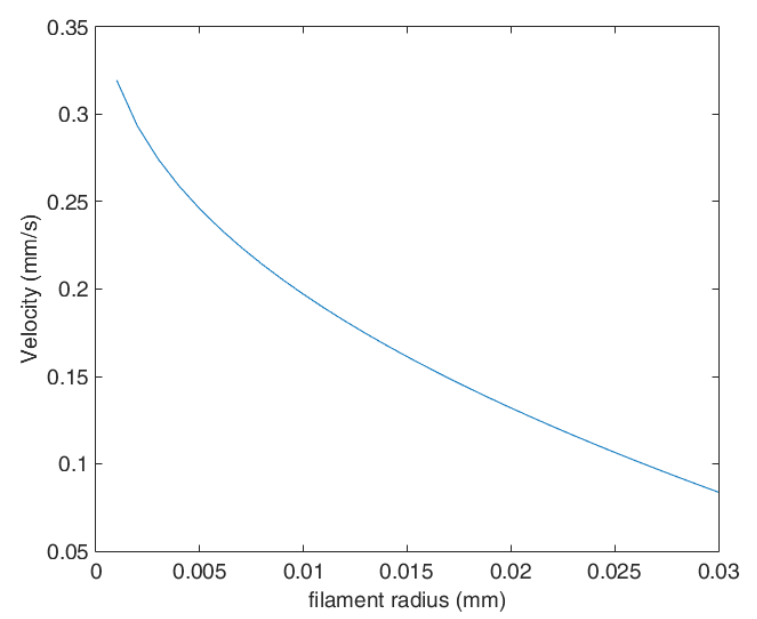

Fig. 5. Relationship between velocity and filament radius

Firstly, the relationship between filament radius and velocity is showed in Fig. 5. With the filament radius increase, the velocity is decrease rapidly. 


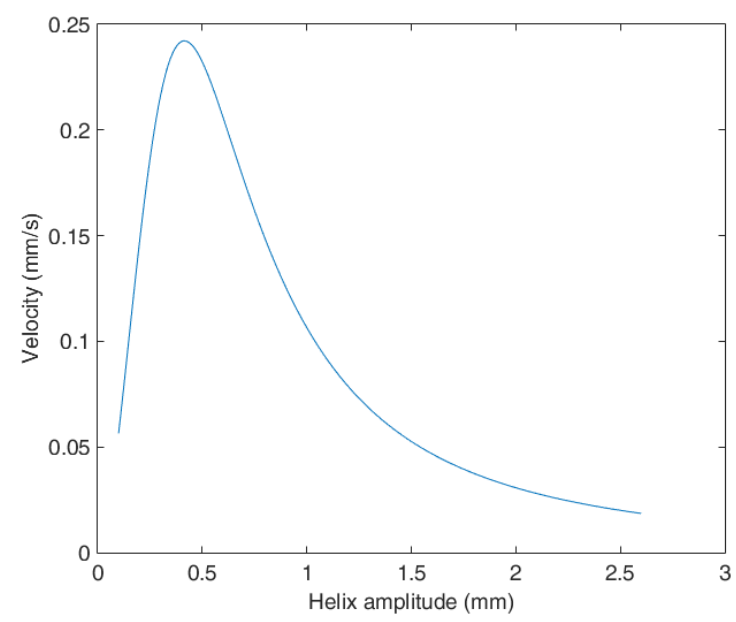

Fig. 6. Relationship between velocity and helix amplitude

Secondly, the relationship between helix amplitude and velocity is illustrated in Fig. 6. From the results, it can be found that there is a maximum value of velocity during the helix amplitude increase. As the helix amplitude continues increases, the velocity shows a decreasing trend.

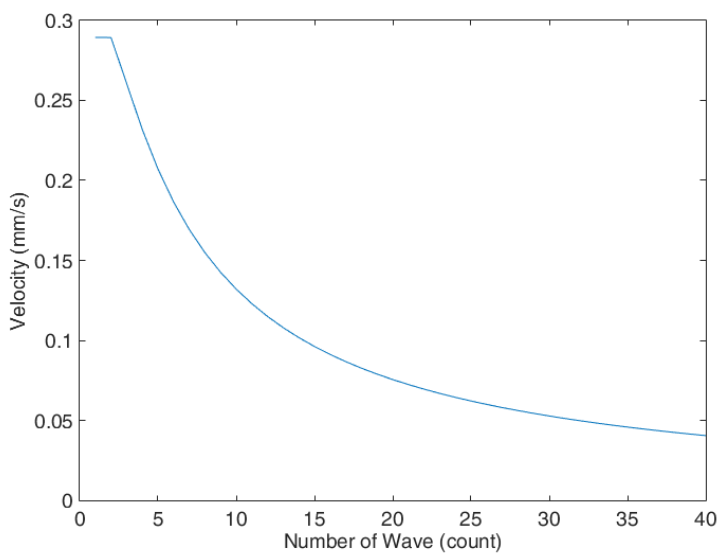

Fig. 7. Relationship between velocity and wave number

Thirdly, the relationship between wavenumber and velocity is showed in Fig. 7. From the graph it is clear that when wave number is respective small, the velocity is keep a stable high value. When the wavenumber increase, the velocity decreased very quickly.

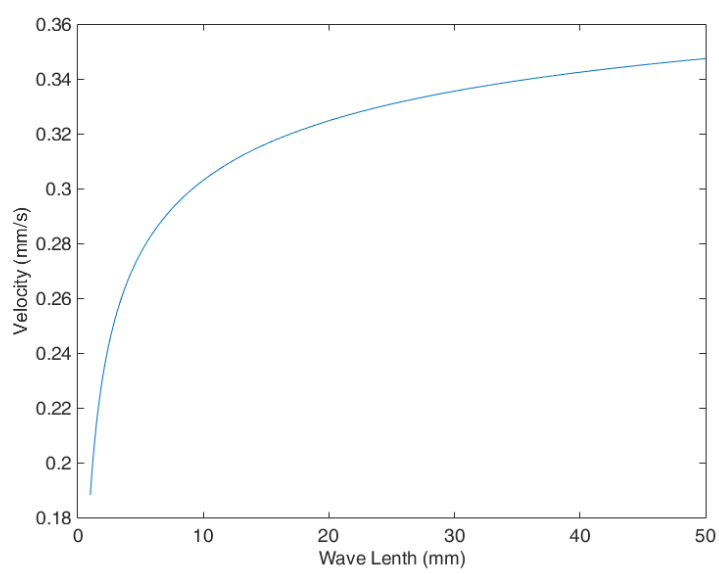

Fig. 8. Relationship between velocity and wavelength

Lastly, the relationship between wavelength $\lambda$ and velocity is illustrated. From the model, it can be predicted that the velocity will increase with the wavelength rising in a specific range, and then keep at a stable level. The dimensionless relationship identified there is a respectively stable value of velocity with wavelength increasing. 
From the analysis above, it is clear that the following strategies need to be consider when fabricate a helix flagella micro swimmer:

The filament radius should be respective small.

The Helix amplitude should set to an optimised value.

The wave number should be set to a small number.

The wave length should be set to a large value.

\section{B. Body Shape Optimisation}

The body shape of artificial micro-swimmer is a rotational ellipsoid as showed in Fig. 9.

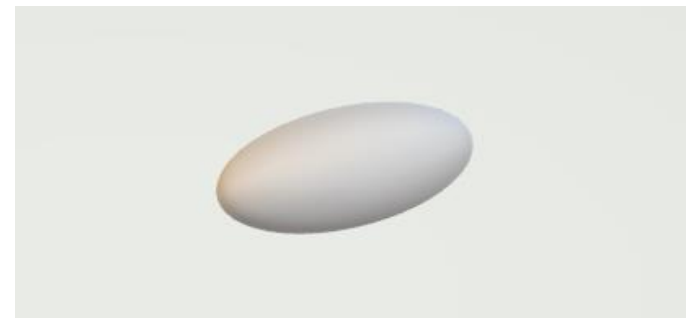

Fig. 9. The shape of body

The length of the long axis equals $a$ and length of short axis equals $b$. The advantage of this shape not only drag convergence caused by streamlined, but also enough space for components like motor, battery etc. The drag applied on the body including viscous force and viscosity moment. The relationships between them and transitory velocity $\mathrm{U}$ and rotation speed $\omega$ of the body are given by (18) and (19).

$$
\begin{aligned}
& F_{b}=C_{F} U \\
& T_{b}=C_{M} \omega
\end{aligned}
$$

Here the coefficient $\mathrm{C}_{\mathrm{F}}$ and $\mathrm{C}_{\mathrm{M}}$ are $3 \times 3$ diagonal matrix given by (20) and (21).

$$
\begin{gathered}
C_{F}=6 \pi \mu a\left(\begin{array}{ccc}
C_{F 3} & * & * \\
* & C_{F 3} & * \\
* & * & C_{F 1}
\end{array}\right) \\
C_{M}=8 \pi \mu a b^{2}\left(\begin{array}{ccc}
C_{M R} & * & * \\
* & C_{M R} & * \\
* & * & C_{M 0}
\end{array}\right)
\end{gathered}
$$

Here $\mathrm{C}_{\mathrm{F} 1}, \mathrm{C}_{\mathrm{F} 3}, \mathrm{C}_{\mathrm{M} 0}$ and $\mathrm{C}_{\mathrm{MR}}$ values are given by (22)-(25) and $\mathrm{e}=\sqrt{\mathrm{a}^{2}+\mathrm{b}^{2}} / a$. From the coefficient relationship above, the viscous force and viscosity moment are related to body shape. So, it is possible to get the drag convergence through optimisation of the body shape.

$$
\begin{gathered}
C_{F 1}=\frac{S e^{3} / 3}{-2 e+\left(1+e^{2}\right) \ln (1+e) /(1-e)} \\
C_{F 3}=\frac{16 e^{3} / 3}{2 e+(-1+3 e 62) \ln (1+e) /(1-e)} \\
C_{M 0}=\frac{4 e^{3} / 3}{2 e-\left(1-e^{2}\right) \ln (1+e) /(1-e)} \\
C_{M R}=\frac{\left(4 e^{3} / 3\right) \times\left(\left(2-e^{2}\right) /\left(1-e^{2}\right)\right)}{-2 e+\left(1+e^{2}\right) \ln (1+e) /(1-e)}
\end{gathered}
$$

If the volume of the inner ellipsoid body is set to a fixed value like $\mathrm{V}=2.8 \times 10^{-5}\left(\mathrm{~m}^{3}\right)$. It is clear that there is only one independent variable about body shape (from the volume calculation formula of ellipsoidal $\mathrm{V}=4 \pi \mathrm{ab}^{3} / 3$ ). It $\mathrm{can}^{\mathrm{be}} \mathrm{set}$ so that the short axis $b$ is the independent variable, then $a=3 \mathrm{~V} / 4 \pi \mathrm{b}^{3}$. The range of values of short axis $b$ is limited by condition $\mathrm{a}>\mathrm{b}$. It is can be defined that $\mathrm{aC}_{\mathrm{F} 1}$ as the first coefficient of drag, $\mathrm{aC}_{\mathrm{F} 3}$ as the second coefficient of drag, $a b^{2} \mathrm{C}_{\mathrm{M} 0}$ as the third coefficient drag, $a b^{3} \mathrm{C}_{\mathrm{MR}}$ as the fourth coefficient of drag. The relation of the coefficients and short axis $b$ is shown in Fig. 10 and Fig. 11. 


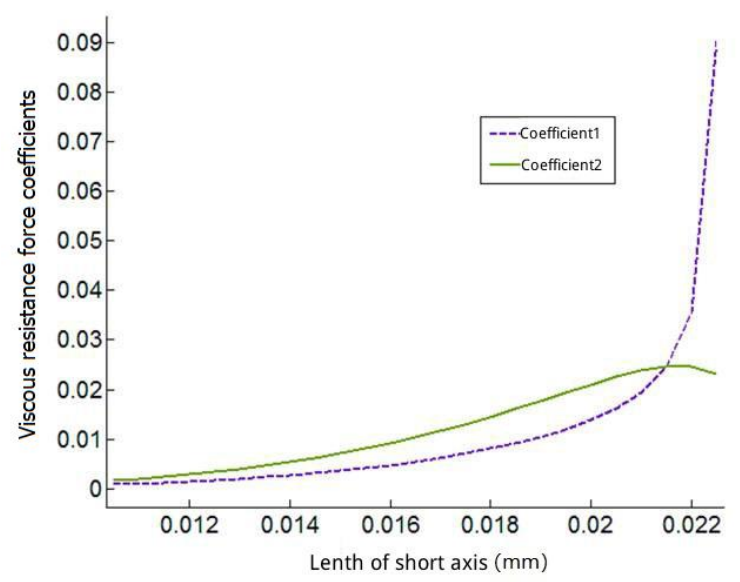

Fig.10. Changing of viscous resistance force coefficients depend on short axis

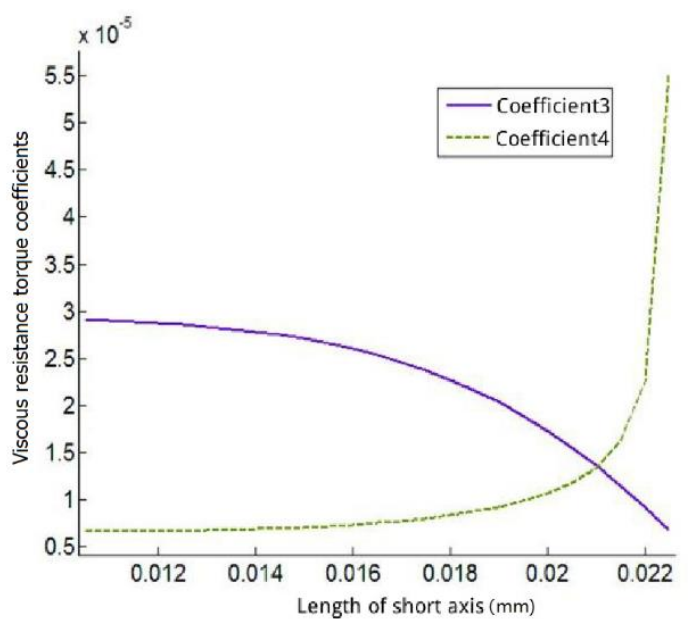

Fig. 11. Changing of viscous resistance torque coefficients depend on short axis

From Fig. 10, it can be found that the viscous resistance force coefficient is going up with the increasing short axis length. But different coefficients have different change trends.

From Fig. 11, it can be found a similar trend with Fig. 10, the resistance torque coefficients grow slowly with short axis size increase, and resistance torque coefficients rise sharply with a certain value of short axis for the third coefficients.

The short axis should be as small as possible when design a realistic robot.

\section{CONCLUSION}

This paper discusses current models and proposes a double-flagellum with a body model for micro swimming robots. It presents Matlab simulation results to analyse the optimum strategies of various parameters e.g. filament radius, helix amplitude, length of short axis etc. Future work can include simulations and comparisons of the proposed double-flagellum with a body model with only-flagellum and single flagellum with a body for various environments.

\section{REFERENCES}

[1] J. Guo, S. Guo, X. Wei, and Q. Gao, “A Novel tele-operation controller for wireless microrobots in-pipe with hybrid motion,” Rob. Auton. Syst., vol. 76, pp. 68-79, 2016.

[2] T. Okada, S. Guo, S. Member, and Y. Yamauchi, "A wireless microrobot with 3 DOFs in pipe for medical applications," in 2011 IEEE/ICME International Conference on Complex Medical Engineering, CME 2011, 2011, pp. 79-84.

[3] C. Flynn, a Taberner, and P. Nielsen, "Modeling the mechanical response of in vivo human skin under a rich set of deformations," Ann Biomed Eng, vol. 39, no. 7, pp. 1935-1946, Jun. 2011.

[4] E. I. Kazakos, J. Kountouras, S. A. Polyzos, and G. Deretzi, "Novel aspects of defensins' involvement in virusinduced autoimmunity in the central nervous system," Med. Hypotheses, vol. 102, pp. 33-36, 2017.

[5] S. Jeong et al., "Penetration of an artificial arterial thromboembolism in a live animal using an intravascular therapeutic microrobot system," Med. Eng. Phys., vol. 38, no. 4, pp. 403-410, 2016.

[6] M. Beckert, B. E. Flammang, E. J. Anderson, and J. H. Nadler, "Theoretical and computational fluid dynamics of an attached remora (Echeneis naucrates)," Zoology, vol. 119, no. 5, pp. 430-438, 2016. 
[7] B. He, Z. Wang, C. Xu, R. Shen, and S. Hu, "The study on pH gradient control in solution for driving bacteria," Biocybern. Biomed. Eng., vol. 33, no. 2, pp. 88-95, 2013.

[8] A. Ghosh, D. Paria, G. Rangarajan, and A. Ghosh, "Velocity Fluctuations in Helical Propulsion: How Small Can a Propeller Be," J. Phys. Chem. Lett., vol. 5, no. 1, pp. 62-68, Jan. 2014.

[9] M. Sauzade, G. J. Elfring, and E. Lauga, "Taylor's swimming sheet: Analysis and improvement of the perturbation series," Phys. D Nonlinear Phenom., vol. 240, no. 20, pp. 1567-1573, 2011.

[10] T. Qiu et al., "Swimming by reciprocal motion at low Reynolds number," Nat. Commun., vol. 5, no. May, pp. 18, 2014.

[11] D. Gonzalez-Rodriguez and E. Lauga, "Reciprocal locomotion of dense swimmers in Stokes flow.," J. Phys. Condens. Matter, vol. 21, no. 20, p. 204103, May 2009.

[12] M. S. Kumar and P. Philominathan, "Purcell's swimmer revisited," Int. J. Autom. Comput., vol. 9, no. 3, pp. 325330, Jun. 2012.

[13] E. Lauga, "Life around the scallop theorem," Soft Matter, vol. 7, no. 7, p. 3060, 2010.

[14] E. M. Purcell, "Life at low Reynolds number,” Am. J. Phys., vol. 45, no. 1, pp. 3-11, Jan. 1977.

[15] J. Lighthill, "Flagellar Hydrodynamics," SIAM Rev., vol. 18, no. 2, p. 161, Apr. 1976.

[16] T. R. Powers, "Role of body rotation in bacterial flagellar bundling," Phys. Rev. E - Stat. Nonlinear, Soft Matter Phys., vol. 65, no. 4, pp. 3-6, Apr. 2002. 